\title{
Enzymatic Antioxidant and Lipid Peroxidation Evaluation in the Newly Diagnosed Breast Cancer Patients in Iran
}

\author{
Parisa Kangari ${ }^{1,2}$, Tahereh Zarnoosheh Farahany ${ }^{3}$, Ali Golchin ${ }^{4 *}$, \\ Somayeh Ebadollahzadeh ${ }^{2}$, Arash Salmaninejad ${ }^{5}$, Soltan Ali Mahboob ${ }^{2}$, \\ Alireza Nourazarian ${ }^{6 *}$
}

\begin{abstract}
Background: Breast cancer is caused by breast tissue malignant cells and it has become one of the main medical concerns with a socio-economic significance especially for women. Among the multiple factors involved in the initiation, progression, and invasion of breast cancer, oxidative stress plays an important role. Antioxidant status, lipid peroxidation, and oxidative stress in newly diagnosed breast cancer patients were determined to find a defined pattern of oxidative stress in these patients. Methods: The malondialdehyde (MDA) levels (as an indicator of lipid peroxidation), glutathione peroxidase (GPX), and superoxide dismutase (SOD) activities of newly diagnosed breast cancer patients $(\mathrm{n}=38)$ and controls $(\mathrm{n}=38)$ were assessed using blood samples. Results: MDA level and SOD activity were significantly higher in the breast cancer patients compared to the healthy subjects group $(\mathrm{p}<0.05)$. Compared to the healthy group, GPX activity decreased significantly in patients group $(\mathrm{p}<0.05)$. Conclusions: High lipid peroxidation is an important risk factor for breast cancer and the increased levels of superoxide anion in breast cancer cells may be a reason for the induction of SOD activity. Nevertheless, oxidative stress is an important factor in development and progression of breast cancer. Further studies on it can lead to a more helpful approach to management of breast cancer.
\end{abstract}

Keywords: Breast cancer- lipid peroxidation- oxidative stress- superoxide dismutase- glutathione peroxidase

Asian Pac J Cancer Prev, 19 (12), 3511-3515

\section{Introduction}

Breast cancer is one of the heterogeneous cancer diseases which is caused by breast tissue malignant cells with diverse clinical symptoms and molecular profiles. The disease causes a serious decline of the quality of life (especially in women) and it has become one of the main medical concerns with a socio-economic significance (Bosch et al., 2010; Shaikh et al., 2017). Nevertheless, and despite its importance, the etiology and pathogenesis are not completely clear. The effects of some of the main effective factors including human races, family history, age, physical activity and obesity, reproductive factors, hormone therapy, and the history of benign breast lesions have been alternately demonstrated in the incidence of breast cancer (Kelsey et al., 1993; Kyu et al., 2016; Newcomb et al., 2011; Reeves et al., 2007; Siegel et al., 2016; Yager and Davidson, 2006). Oxidative stress, that is an imbalance between oxygen free-radical generation and antioxidant scavenging, plays an important role in the initiation, progression, and invasion of breast cancer. Excessive formation of oxygen free radicals can cause oxidative damage to biomolecules and consequently result in mutagenesis, lipid peroxidation, and carcinogenesis (Hecht et al., 2016; Yue and Wang, 2015). The prime targets of reactive oxygen species (ROS) are the polyunsaturated fatty acids (PUFA) and some of the proteins found in cell membrane (Stanicka et al., 2015). Additionally, impaired lipid hydro-peroxides yields a wide range of end-products including malondialdehyde (MDA). On the other hand, lipid peroxidation induced by oxygen-free-radical can led to malignant transformation (Aldini, 2010; Halliwell, 2007; Nechuta et al., 2014). Several studies have shown that oxidative stress increases in breast cancer (Balliet et al., 2011; Fazilaty et al., 2013; Gönenç et al., 2005; Halliwell, 2007). 
The molecular mechanism is the most widely discussed subject among the topics of cancer research and therapy (Mishra et al., 2018). Antioxidants are defense mechanisms against oxidative stress that remove reactive species and sustain redox status. The most important enzymatic antioxidants are catalase, glutathione peroxidase (GPX), and superoxide dismutase (SOD); and non-enzymatic antioxidants are found in vitamin $\mathrm{E}$, vitamin $\mathrm{C}$, vitamin $\mathrm{A}$, flavonoids, albumin, glutathione, thioredoxins, uric acid, metabolites of polyphenols, and metal ions chelators such as ferritin and transferrin (Gönenç et al., 2006; Hecht et al., 2016; Hou et al., 2003; Lopaczynski and Zeisel, 2001). It is believed that the antioxidant properties protect various cells from oxidative damage by several pathways so that in each pathway especial enzymes and molecules have distinctive role (Sharifi Rad et al., 2014; Varoni et al., 2016).

The oxidative stress state was evaluated by measuring MDA as an indicator of lipid peroxidation and SOD and GPX as the indicator of antioxidant in the patients with breast cancer to achieve a defined pattern of oxidative stress in breast cancer pathophysiology. The results can be useful as diagnostic and therapeutic finding for the patients (Figure 1).

\section{Materials and Methods}

Informed consent was obtained according to institutional guidelines as per the Declaration of Helsinki Principles. The study was approved by Local Ethics Committee, Tabriz University of Medical Science.

\section{Inclusion criteria}

Totally 38 patients with newly diagnosed breast cancer (mean age: $40.78 \pm 0.847$ years - Noor Nejat Hospital of Tabriz, Iran) as case group and 38 healthy women (mean age: $39.61 \pm 0.927$ years) as control group enrolled in the study. Informed consent was signed by each patient before enrollment. None of the patients and controls had concomitant diseases such as diabetes mellitus, liver disease, nephrotic syndrome, hypertension, or previous history of breast cancer and none of them used contraceptives, oral medications, hormones, or vitamins. In addition, none of them drank or smoked. The patients were not on chemotherapy and radio therapy before sampling and the both patients and controls were pre-menopausal. Breast cancer patients were classified using TNM system.

\section{Blood sampling}

Peripheral venous blood samples were taken into tubes after an overnight fasting for at least 10 hours. To measure MDA, the blood samples were centrifuged at $3000 \mathrm{rpm}$ for 5 minutes, and the serum was separated at room temperature $\left(25^{\circ} \mathrm{C}\right)$. The activities of SOD and GPX were determined for all blood samples. The prepared samples were immediately frozen in aliquots at $-80{ }^{\circ} \mathrm{C}$ and stored until analysis.

\section{Determination of MDA}

Serum MDA level was determined through thiobarbituricacid (TBA) method. The serum (500 $\mu \mathrm{L})$ was mixed with $1 \mathrm{~mL}$ of TBA $(0.67 \%)$ and $3 \mathrm{~mL}$ of phosphoricacic (1\%) and then placed in bathroom for $45 \mathrm{~min}$ (Kumari and Menon, 1987). After cooling, the products were extracted in $3 \mathrm{ml}$ of normal butanol and centrifuged at $3,000 \mathrm{rpm}$ at $\left(4{ }^{\circ} \mathrm{C}\right)$ for $10 \mathrm{~min}$ and the absorbance was measured by spectrophotometer at $532 \mathrm{~nm}$.

\section{Determination of SOD activity}

SOD activity was measured through spectrophotometric method, using commercial Ransod kit (Randox Laboratories, UK) and autoanalyzer (Alcyon300Abbot). This method employs xanthine and xanthine oxidase (XOD) to generate superoxide radicals that react with 2-(4-iodophenyl)-3-(4-nitrophenol)-5phenyltetrazoliumchloride (I.N.T) to form a red formazan dye. The SOD activity was then measured by the degree of inhibition of this reaction. One unit of SOD is an amount of SOD that causes $50 \%$ inhibition of the rate of reduction of INT under the conditions of the assay. The results were articulated as U/g Hb (Misra and Fridovich, 1972).

\section{Determination of GPX activity}

GPX activity was assessed spectrophotometrically using Ransod kit (Randox Laboratories, UK) and autoanalyzer (Alcyon300abbot). This method is based on Paglia-Valentine method. GPX catalyses the oxidation of Glutathione (GSH) by Cumene Hydroperoxide. The results were expressed by $\mathrm{U} / \mathrm{g} \mathrm{Hb}$ (Flohé and Günzler, 1984).

\section{Statistical analysis}

Based on sample size formula for comparing the mean scores between two groups, the sample size (with $95 \%$ confidence and $95 \%$ power) was obtained equal to 38 patients (with Breast cancer) and 38 controls. The participants were selected using random sampling. The data were analyzed using SPSS version 11.5 and normality was tested by Q-Q test for all continuous variables. Outlier data was removed and ANOVA with control covariate was used for controlling confounding variables between the two groups in analysis design. Error bar was used for showing confidence interval and deference and relationship between them. All the results were expressed as Mean $\pm \mathrm{SE}$ and the level of significance was set at $\mathrm{P}$-value $<0.05$.

\section{Results}

The main demographic characteristics of the two groups are presented in Table 1 . The results show lipid peroxidation and antioxidants status in blood samples of breast cancer patients and controls (Table 2). The MDA levels, as an indicator of lipid peroxidation, were significantly increased in the patients as compared with the controls $(\mathrm{P}=0.000)$. The mean levels of MDA were $3.77 \pm 0.25 \mathrm{nmol} / \mathrm{ml}$ and $1.92 \pm 0.12 \mathrm{nmol} / \mathrm{ml}$ in the patient and control groups respectively (Figure 2). In addition, SOD activity was significantly increased in the breast cancer subjects $(\mathrm{P}=0.004)$ compared with controls. The 


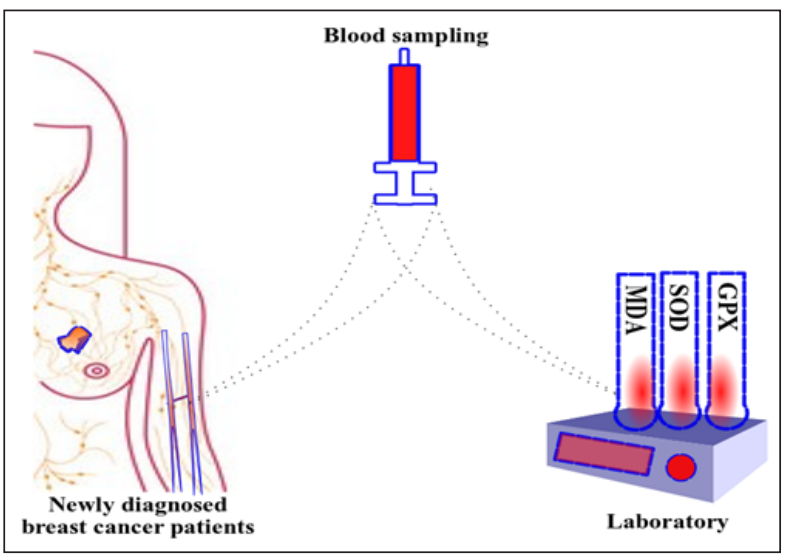

Figure 1. Study Design which Shows the Summary of the Study Protocol

Table 1. General Characteristics of Breast Cancer Patients and Controls

\begin{tabular}{lcc}
\hline General characteristics & Breast cancer & Controls \\
\hline 1.Totalnumberof subjects & 38 & 38 \\
2.Age (mean \pm SE years) & $40.78 \pm 0.847$ & $39.61 \pm 0.927$ \\
3. Age at Menarche & $15 \pm 2$ & $14 \pm 2$ \\
4.Menopausal status & & \\
$\quad$ Premenopausal & $(38) 100 \%$ & $(38) 100 \%$ \\
$\quad$ Postmenopausal & - & - \\
5.Clinical stage & & - \\
Stage I & 8 & - \\
Stage II & 18 & - \\
Stage III & 8 & - \\
Stage IV & 4 & - \\
\hline
\end{tabular}

SOD activity was $1737.5 \pm 51.19 \mathrm{U} / \mathrm{gHb}$ for the breast cancer subjects and $1539.47 \pm 43.71 \mathrm{U} / \mathrm{gHb}$ for the controls (Figure 3a). On the other hand, the GPX activity was significantly lower in the breast cancer patients in comparison with the controls $(\mathrm{P}=0.001)$. In the patient and control groups, the GPX activity was $62.19 \pm 1.70$ $\mathrm{U} / \mathrm{gHb}$ and $71.05 \pm 1.82 \mathrm{U} / \mathrm{gHb}$, respectively (Figure $3 \mathrm{~b}$ ).

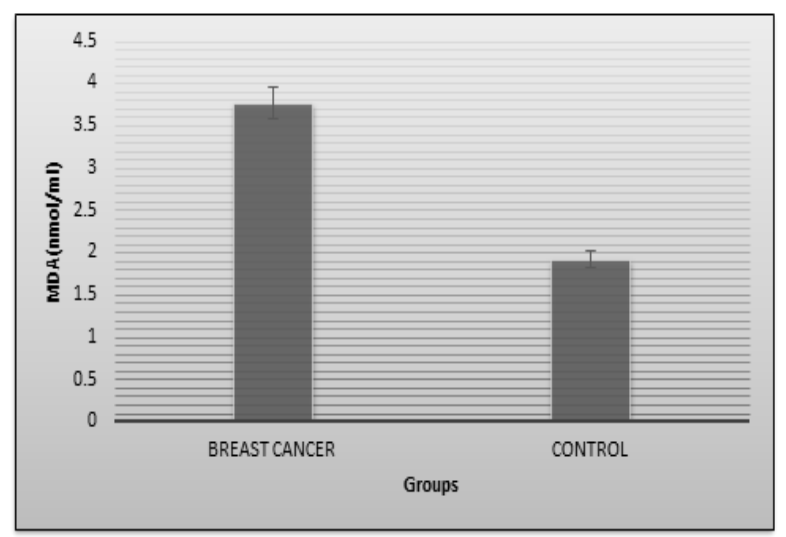

Figure 2. Extent of Lipid Peroxidation (MDA) in Serum of Patients and Controls. The bar diagram represents level of MDA in controls and cases as mean \pm SE of three individual experiments done in triplicates
Antioxidant Activity in Breast Cancer Patients

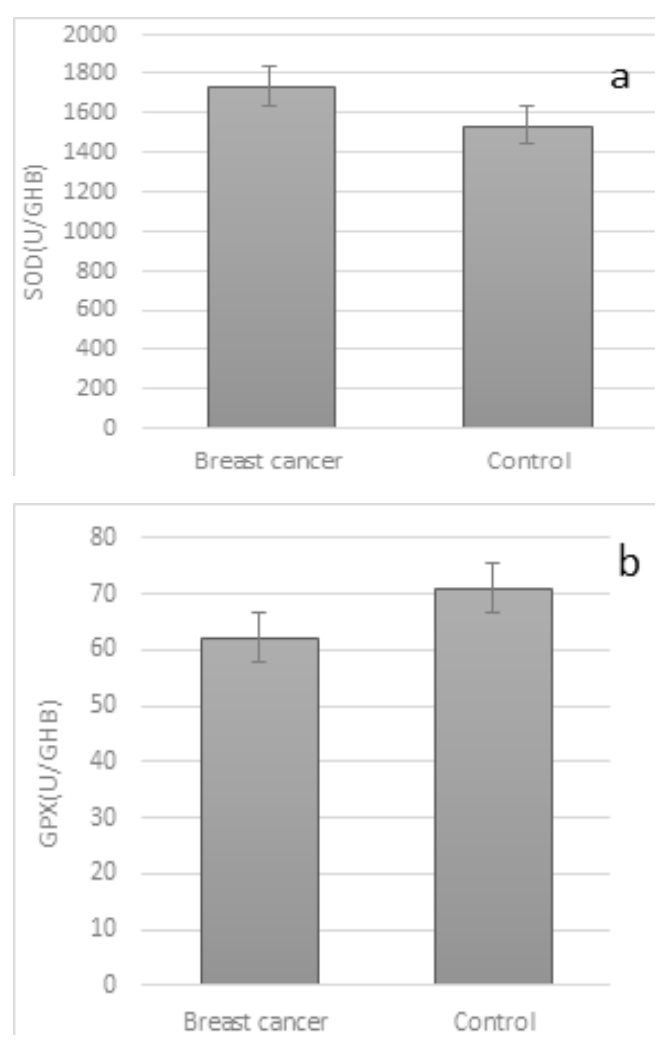

Figure 3. a) SOD activity, b) GPX activity, in patients and controls. The bar diagram represents activity of SOD and GPX in controls and cases as mean $\pm \mathrm{SE}$ of three individual experiments done in triplicates

Table 2. Levels of Lipid Peroxidation and Antioxidants Status in Blood of Breast Cancer Patients and Controls

\begin{tabular}{lcc}
\hline Parameter & $\begin{array}{c}\text { Breast cancer patient } \\
(\mathrm{n}=38)\end{array}$ & $\begin{array}{c}\text { Controls } \\
(\mathrm{n}=38)\end{array}$ \\
\hline MDA $(\mathrm{nmol} / \mathrm{ml})$ & $3.77 \pm 0.25$ & $1.92 \pm 0.12$ \\
SOD $(\mathrm{U} / \mathrm{gHb})$ & $1737.5 \pm 51.19$ & $1539.47 \pm 43.71$ \\
$\mathrm{GPX}(\mathrm{U} / \mathrm{gHb})$ & $62.19 \pm 1.70$ & $71.05 \pm 1.82$ \\
\hline
\end{tabular}

\section{Discussion}

Oxidative stress is an important factor in cancer devolvement and a key factor for disease evolution (Tuma, 2002). Previous studies have confirmed increased oxidative stress in different types of cancer, including breast cancer (Abdel-Salam et al., 2011). Anticipated mechanisms for amplified oxidative stress in breast cancer is assumed to have the ability to induce genetic changes in antioxidant enzymes, estrogen treatment, intemperance generation of reactive oxygen species, as well as impaired antioxidant system (Sreenivasa Rao et al., 2012). There are abundant evidences demonstrating a contributing role of free radicals, oxidative damage, and lipid peroxidation in occurrence and development of the cancer types (Czerska et al., 2015; Gupta et al., 2012; Hauck and Bernlohr, 2016). The most general method to evaluate oxidative stress is to measure the compounds obtained by the reaction of oxidants with biomolecules as a biomarker (Gupta et al., 2012; Sreenivasa Rao et al., 2012). The biomarkers of 
oxidative stress are clinically important and evaluation of body tissue and blood is away to identify cancers such as breast cancer (Czerska et al., 2015; Hauck and Bernlohr, 2016).

Several studies have indicated that lipid peroxidation may be involved in tumor promotion because this process can generate reactive and toxic metabolites. As one of the most abundant and important aldehydes of lipid peroxidation, MDA can react with proteins, DNA, and other biomolecules and thereby alter their structure and function. In recent years, assessment of MDA of tissues and plasma has been extensively used in numerous cancers including breast cancer. Various studies have examined the possibility of a relationship between lipid peroxidation and breast cancer (Gonenc et al., 2006a; Gönenç et al., 2006b; Hauck and Bernlohr, 2016; Savini et al., 2016; Sreenivasa Rao et al., 2012). Some studies have reported higher serum MDA levels in breast cancer patients (Aldini et al., 2010; Savini et al., 2016) while some have reported lower levels (Abdel-Salam et al., 2011; Gerber et al., 1996; Gonenc et al., 2006; Tas et al., 2005). Our findings supported the common observation that breast malignancies are related to an increased level of MDA. The results showed that serum levels of MDA are considerably increased in breast cancer as compared to healthy subjects. Since our patients group was formed from newly diagnosed breast cancer patients, the increased level of oxidative factors can be a feature of the early stages of cancer progression. Increased MDA levels in the serum of breast carcinoma may be in response to the aggregation of ROS, which might result in extensively elevated cellular lipid peroxidation (Abdel-Salam et al., 2011; Tas et al., 2005).

Moreover, SOD catalyzes the conversion of superoxide anion radical $\left(\mathrm{O}^{\circ}{ }^{\circ}\right)$ into an oxygen molecule and a hydrogen peroxide (Stepp et al., 2014). In the present study, SOD activity was significantly increased in the breast cancer patients as compared with the healthy subjects. In contrast, the findings of Gupta et al., (2012) suggested decreased SOD activity in breast cancer patients compared with healthy subjects. Studies have indicated that oxidative surroundings in tumor may inflame oxidants and activate gene expression through the antioxidant responsive elements (ARE) (Czerska et al., 2015; Hauck and Bernlohr, 2016), which may result in an enhanced SOD activity. GPX is a glycoprotein comprised of a single seleno-cysteine residue at the active site of each subunit. To protect biological organisms from oxidative damage, GPX catalyzes the reduction of hydrogen peroxide and lipid hydroperoxides to water and the related alcohols (Jomova et al., 2011). Some studies have illustrated an abundance of GPX activity in erythrocytes of the patients with breast carcinoma (Sreenivasa Rao et al., 2012). In addition, there are reports of increased GPX activity in breast tumors (Wang et al., 2014). However, Gupta et al., (2012) reported decreased GPX activity in women with breast cancer. Our findings showed a reduction in GPX activity in breast cancer as compared to healthy controls, which is consistent with the earlier studies. Decreased GPX activity may lead to accumulation of the reactive species of hydrogen peroxide.
The results showed that the increased production of superoxide anion probably induces higher superoxide dismutase activity in breast cancer patients, which is responsible for the dis-mutation of the $\mathrm{O}_{2}^{\circ}$ into $\mathrm{H}_{2} \mathrm{O}_{2}$. On the other hand, decreased glutathione peroxidase activity leads to accumulation of hydrogen peroxide. However, there may be a gathering of $\mathrm{H}_{2} \mathrm{O}_{2}$ consequential to elevated production of the $\mathrm{OH}$ radical, which was supported by the higher lipid peroxidation and increased production of MDA contents. In Conclusion, breast cancer is associated with enhancement of lipid peroxidation in plasma with affiliated decrease of antioxidant defense capacity. These results refer to an increased oxidative stress in those patients. Elevated lipid peroxidation and oxidative stress are leading causes of breast cancer progression. Generally, our findings sustain the significance of endogenous antioxidant in the etiology of breast cancer across all levels of predicted risk.

\section{Conflict of interests}

Competing interests All authors declare that they have no conflict of interests.

\section{Acknowledgments}

The authors wish to thank the patients for participating in the study. They are also grateful to Drug Applied Research Center, Tabriz University of Medical Science for their collaboration. This study is a part of MSc. dissertation by Parisa Kangari conducted from 2014 to 2016.

\section{References}

Abdel-Salam OME, Youness ER, Hafez HF (2011). The antioxidant status of the plasma in patients with breast cancer undergoing chemotherapy. Open J Mol Integr Physiol, 1, 29-35.

Aldini G, Yeum KJ, Niki E, Russell R (2010). Biomarkers for antioxidant defense and oxidative damage: principles and practical applications. Wiley-Blackwell, USA pp.xv +363 pp. ref.many.

Balliet RM, Capparelli C, Guido C, et al (2011). Mitochondrial oxidative stress in cancer-associated fibroblasts drives lactate production, promoting breast cancer tumor growth. Cell Cycle, 10, 4065-73.

Bosch A, Eroles P, Zaragoza R, et al (2010). Triple-negative breast cancer: Molecular features, pathogenesis, treatment and current lines of research. Cancer Treat Rev, 36, 206-15.

Czerska M, Mikołajewska K, Zieliński M, Gromadzińska J, Wąsowicz W (2015). Today's oxidative stress markers. Med Pr, 66, 393-405.

Fazilaty H, Gardaneh M, Bahrami T, Salmaninejad A, Behnam B (2013). Crosstalk between breast cancer stem cells and metastatic niche: emerging molecular metastasis pathway?. Tumor Biol, 34, 2019-30.

Flohé L, Günzler WA (1972). Assays of glutathione peroxidase. Methods Enzymol, 105, 114-21.

Gerber M, Astre C, Ségala C, et al (1996). Oxidant-antioxidant status alterations in cancer patients: Relationship to tumor progression. J Nutr, 126, 1201-7.

Gönenç A, Erten D, Aslan S, et al (2006a). Lipid peroxidation and antioxidant status in blood and tissue of malignant breast tumor and benign breast disease. Cell Biol Int, 30, 376-80. 
Gönenç A, Tokgöz D, Aslan S, Torun M (2005). Oxidative stress in relation to lipid profiles in different stages of breast cancer. Indian J Biochem Biophys, 42, 190-4.

Gönenç A, Erten D, Aslan S, et al (2006b). Lipid peroxidation and antioxidant status in blood and tissue of malignant breast tumor and benign breast disease. Cell Biol Int, 30, 376-80.

Gupta RK, Patel AK, Kumari R, et al (2012). Interactions between oxidative stress, lipid profile and antioxidants in breast cancer: a case control study. Asian Pac J Cancer Prev, 13, 6295-8.

Halliwell B (2007). Oxidative stress and cancer: have we moved forward?. Biochem J, 401, 1-11.

Hauck AK, Bernlohr DA (2016). Oxidative stress and lipotoxicity. J Lipid Res, 57, 1976-86.

Hecht F, Pessoa CF, Gentile LB, et al (2016). The role of oxidative stress on breast cancer development and therapy. Tumor Biol, 37, 4281-91.

Hou MF, Lin S Bin, Yuan SSF, et al (2003). The clinical significance between activation of nuclear factor kappa B transcription factor and overexpression of HER-2/neu oncoprotein in Taiwanese patients with breast cancer. Clin Chim Acta, 334, 137-44.

Jomova K, Jenisova Z, Feszterova M, et al (2011). Arsenic: toxicity, oxidative stress and human disease. J Appl Toxicol, 31, 95-107.

Kelsey JL, Gammon MD, John EM (1993). Reproductive factors and breast cancer. Epidemiol Rev, 15, 36-47.

Kumari SS, Menon VP (1987). Changes in levels of lipid peroxides and activities of superoxide dismutase and catalase in isoproterenol induced myocardial infarction in rats. Indian J Exp Biol, 25, 419-23.

Kyu HH, Bachman VF, Alexander LT, et al (2016). Physical activity and risk of breast cancer, colon cancer, diabetes, ischemic heart disease, and ischemic stroke events: systematic review and dose-response meta-analysis for the Global Burden of Disease Study 2013. BMJ, 354, 3857.

Lopaczynski W, Zeisel SH (2011). Antioxidants, programmed cell death, and cancer. Nutr Res, 21, 295-307.

Mishra AP, Salehi B, Sharifi-Rad M, et al (2018). Programmed cell death, from a cancer perspective: An overview. $\mathrm{Mol}$ Diagn Ther, 22, 281-95.

Misra HP, Fridovich I (1972). The role of superoxide anion in the autoxidation of epinephrine and a simple assay for superoxide dismutase. J Biol Chem, 247, 3170-5.

Nechuta S, Cai Q, Zheng Y, et al (2014). Urinary biomarkers of oxidative stress and breast cancer survival. Cancer Causes Control, 25, 701-7.

Newcomb PA, Trentham-Dietz A, Hampton JM, et al (2011). Late age at first full term birth is strongly associated with lobular breast cancer. Cancer, 117, 1946-56.

Reeves GK, Pirie K, Beral V, et al (2007). Cancer incidence and mortality in relation to body mass index in the Million Women Study: cohort study. BMJ, 335, 1134-34.

Savini I, Gasperi V, Catani MV (2016). Oxidative stress and obesity; in: Obesity. Cham, Springer International Publishing, pp 65-86.

Shaikh S, Channa NA, Talpur FN, et al (2017). Radiotherapy improves serum fatty acids and lipid profile in breast cancer. Lipids Health Dis, 16, 92.

Sharifi Rad J, Hoseini Alfatemi SM, Sharifi Rad M, Iriti M (2014). Free radical scavenging and antioxidant activities of different parts of Nitraria schoberi L. J Biol Act Prod Nat, 4, 44-51.

Siegel RL, Miller KD, Jemal A (2016). Cancer statistics, 2016. CA Cancer J Clin, 66, 7-30.

Sreenivasa Rao CS, Sarala Kumari D, Kumari DS (2012). Changes in plasma lipid peroxidation and the antioxidant
Antioxidant Activity in Breast Cancer Patients

system in women with breast cancer. Int J Basic Appl Sci, 1, 429-38.

Stanicka J, Landry W, Cotter TG (2015). Oxidative stress biomarkers and ROS molecular probes. Oxidative Stress: Diagnostics, Prevention, and Therapy, 2, pp 353-74.

Stepp MW, Folz RJ, Yu J, Zelko IN (2014). The c10orf10 gene product is a new link between oxidative stress and autophagy. Biochim Biophys Acta - Mol Cell Res, 1843, 1076-1088.

Tas F, Hansel H, Belce A, Ilvan S, et al (2005). Oxidative Stress in Breast Cancer. Med Oncol, 22, 11-16.

Tuma DJ (2002). Role of malondialdehyde-acetaldehyde adducts in liver injury. Free Radic Biol Med, 32, 303-8.

Varoni EM, Lo Faro AF, Sharifi-Rad J, Iriti M (2016). Anticancer molecular mechanisms of resveratrol. Front Nutr, 3, 8.

Wang C, Yu J, Wang H, Zhang J, Wu N (2014). Lipid peroxidation and altered anti-oxidant status in breast adenocarcinoma patients. Drug Res (Stuttg), 64, 690-2.

Yager JD, Davidson NE (2006). Estrogen carcinogenesis in breast cancer. $N$ Engl J Med, 354, 270-82.

Yue W, Wang J-P (20155). Estrogen metabolites and breast cancer. Steroids, 99, 61-6.

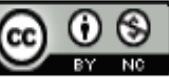

This work is licensed under a Creative Commons AttributionNon Commercial 4.0 International License. 